\title{
Balkanologie
}

Balkanologie Revue d'études pluridisciplinaires

Vol. X, $n^{\circ} 1-2 \mid 2008$

Volume $X$ Numéro 1-2

\section{Fronts et frontières dans les Balkans : les géographes et les enjeux frontaliers sur le Danube en 1919-1920}

Emmanuelle Boulineau

\section{OpenEdition}

Journals

Édition électronique

URL : http://journals.openedition.org/balkanologie/396

DOI : 10.4000/balkanologie.396

ISSN : 1965-0582

Éditeur

Association française d'études sur les Balkans (Afebalk)

Référence électronique

Emmanuelle Boulineau, « Fronts et frontières dans les Balkans : les géographes et les enjeux frontaliers sur le Danube en 1919-1920», Balkanologie [En ligne], Vol. X, n 1-2 | 2008, mis en ligne le 03 juin 2008, consulté le 17 décembre 2020. URL : http://journals.openedition.org/balkanologie/396 ; DOI : https://doi.org/10.4000/balkanologie.396

Ce document a été généré automatiquement le 17 décembre 2020.

(c) Tous droits réservés 


\title{
Fronts et frontières dans les Balkans : les géographes et les enjeux frontaliers sur le Danube en 1919-1920
}

\author{
Emmanuelle Boulineau
}

\begin{abstract}
« Les conflits étaient particulièrement vifs à propos des îles : si le thalweg passait au nord d'une île, elle devenait sur l'heure bulgare et les habitants des villages voisins se précipitaient en barques ou en plates, pour y couper les saules et les peupliers et y faucher le foin. Les Roumains protestaient, ils rappliquaient de leurs villages [...]. Mais voilà qu'avant que le conflit n'ait pu être réglé, le Danube modifiait son cours, le thalweg se rabattait vers notre rive et les Roumains triomphants s'emparaient de leur île avec des haches pour y couper la forêt. »

Yordan Raditchkov

Sur l'eau
\end{abstract}

Une carte d'Europe aujourd'hui montre l'importance du Danube, long de quelque 2860 $\mathrm{km}$, et support de frontière, ce trait rouge épais des cartes politiques. Surimposée par la cartographie sur les éléments du relief, la ligne frontalière épouse dans les Balkans les contours du fleuve sur de vastes segments : le Danube porte $470 \mathrm{~km}$ de frontière entre la Bulgarie et la Roumanie, $344 \mathrm{~km}$ entre la Roumanie et l'Ukraine, $290 \mathrm{~km}$ entre la Roumanie et la Serbie-Monténégro et un peu moins de 200 km entre cette dernière et la Croatie soit environ $1300 \mathrm{~km}$ et quasiment la moitié de sa longueur totale. L'impression donnée par la carte justifie l'appellation du Danube comme frontière naturelle, gravée dans la longue durée. Mais c'est oublier, d'une part, que les cartes mentent ${ }^{1}$ et, d'autre part, que l'on ne se baigne jamais deux fois dans le même fleuve, comme nous l'enseigne Héraclite depuis bien des siècles...

Les cartes mentent par omission - le procédé cartographique exige une sélection d'informations-, par manque de précision -cette information est réduite- ou par manipulation - selon ce que veut dire l'auteur par la carte. Et pour preuve : la fixité du trait fluvial sur la carte fait oublier la fluidité du cours d'eau dans la réalité. L'idée de frontière fluviale renforce ce trait: à la recherche de la ligne continue et stable 
repérable sur la carte, le fleuve oppose sur le terrain des eaux mouvantes et divagantes. Étudier le Danube comme support de frontière dans les Balkans conduit nécessairement à s'interroger sur la frontière naturelle, celle qui allie élément de la nature et construction politique, et sur la recherche d'une bonne frontière, dans la hantise d'en tracer une mauvaise. Pour ce faire, nous avons choisi un moment, des lieux et des acteurs, avec pour ambition de relire des écrits historiques à la lumière d'une approche d'épistémologie de la géographie.

Le moment, 1919-1920, correspond au tracé des frontières dans cette Europe balkanique qui sort du premier conflit mondial. De nombreuses frontières sont à inventer, elles consacrent la naissance ou le remaniement des États balkaniques sur les ruines des empires. Il s'agit d'un moment rare de remise en question des frontières sur la carte d'Europe qui se déroule à la Conférence de la paix de Paris. La question des fleuves comme porteur de frontière se pose alors avec une grande acuité. On place ainsi notre réflexion sur le Danube au miroir de la question rhénane contemporaine. L'hypothèse d'une distorsion entre la connaissance du terrain et la ligne frontière imaginaire ou imaginée guide notre étude pour comprendre comment tracer la frontière. Elle engage une réflexion géographique sur les échelles d'analyse envisagées et les types d'enjeux. Nous chercherons ainsi à relativiser l'importance de l'argument de la frontière naturelle pour expliquer le choix des tracés aux abords du fleuve.

Les lieux explorés dans ce travail font appel au terrain, à la réalité du tracé et de la démarcation de la frontière, à la connaissance de ces espaces balkaniques. Nous avons analysé les expertises produites pour tracer la frontière dans trois espaces bordiers du Danube: la plaine panonienne sud, le Banat et la Dobroudja afin d'envisager la distorsion entre les impératifs politiques du tracé des frontières lors de la Conférence de la paix et l'appréhension géographique d'un terrain à baliser.

Enfin, parmi les multiples acteurs engagés dans le tracé des frontières en 1919-1920, nous avons retenu les géographes parmi les experts scientifiques mandatés par les gouvernements à l'occasion de la Conférence de Paris. C'est l'occasion de croiser leurs regards avec celui des historiens, linguistes, militaires et de s'attacher à l'argumentaire développé. L'intérêt pour les géographes experts permet d'étudier ainsi la mobilisation des savoir-faire et des notions propres à une discipline universitaire en cours de consolidation et encore tournée vers l'action. Le champ des expertises envisagées se limite aux sources européennes et françaises essentiellement. Les expertises américaines, l'étude de la posture de l'expert et de ses réseaux d'inscription sont écartées de notre propos, par souci de brièveté et par conscience que l'approche y gagnerait davantage par une démarche interdisciplinaire.

Tracer la frontière en 1919-1920 s'inscrit dans un ordre juridique, celui de la souveraineté de jeunes États modernes sur leur territoire, et dans un ordre politique, le contexte historique de la fin de la Première Guerre mondiale. Si le dessin de la frontière s'inscrit ainsi dans un contexte diplomatique particulier, il engage, pour les experts, une autre temporalité, celle de la territorialisation des identités nationales et des entités étatiques, que l'on sait fortement problématique dans le monde balkanique.

\section{Fleuve-frontière, le Danuble au miroir du Rhin}

Regarder le Danube au miroir du Rhin, c'est explorer la charge symbolique de deux fleuves européens porteurs de mythes et de légendes, sources d'inspiration de grandes 
œuvres littéraires, artères vitales d'une Europe mondialisée. La comparaison entre la question rhénane et la question danubienne lors de la Conférence de la Paix qui débute en 1919 à Paris est un projet ambitieux que nous ne pouvons pas conduire dans ces lignes. Notre projet se limite à une mise en perspective de la réflexion sur les enjeux frontaliers autour du Danube par rapport à la question rhénane, afin de faire ressortir les conceptions du fleuve comme support de frontière en Europe balkanique. Les sources nous y autorisent car la réflexion préparatoire sur les frontières s'appuie en grande partie sur le rôle du Rhin et du Danube dans la future Europe. Côté français, les membres du comité d'études, formé dès 1917 à l'initiative du gouvernement pour réfléchir aux conséquences territoriales de la guerre, ont produit des expertises publiées dans deux abondants volumes accompagnés d'un atlas respectif ${ }^{2}$. C'est l'occasion d'étudier la définition de la frontière au début du XXe siècle.

Le premier volume, consacré à l'Alsace-Lorraine et aux frontières du Nord-Est, rend compte d'une question territoriale cruciale pour la France - le recouvrement d'une province perdue. La question rhénane occupe une place importante dans ces expertises: près des deux tiers du volume y sont consacrés, notamment dans deux parties traitant l'une du Rhin comme fleuve international ( $4^{\mathrm{e}}$ partie), l'autre des populations rhénanes $\left(5^{\mathrm{e}}\right.$ partie). Les géographes participent activement à la réflexion sur le Rhin: Emmanuel de Martonne s'attache aux enjeux économiques et aux conditions physiques de la navigation sur le fleuve ${ }^{3}$, Lucien Gallois publie un court texte sur le potentiel hydro-électrique du fleuve ${ }^{4}$. Le Danube n'est pas absent de ce volume puisque le général Bourgeois, chef du Service géographique des Armées, présente une note sur la commission de navigation du Danube, à la suite de sa présentation de la navigation sur le Rhin ${ }^{5}$. Dans le second volume des travaux du comité d'études, la part accordée au Danube est bien plus réduite puisqu'une seule intervention y est consacrée : le général Bourgeois étudie là encore la nécessité de garantir la liberté et la neutralité de navigation sur le fleuve ${ }^{6}$. En revanche, le Danube apparaît en creux dans de nombreuses études consacrées, dans la partie balkanique, aux frontières de la YougoSlavie et de la Roumanie.

Les titres des interventions infirme l'idée d'une mobilisation de la notion de frontière naturelle sous la plume des experts. La recension des occurrences du terme de «frontière naturelle " donne des résultats bien maigres. En évoquant la frontière du Nord-Est de 1871, Lucien Gallois concède que «nulle part, sauf dans les Vosges, cette limite ne correspondait à des obstacles naturels. La France était ouverte [... $]^{7}$ ». Mais la formule porte sur la montagne vosgienne comme support de frontière. On retrouve en fait toute l'ambiguïté de la frontière naturelle que la question fluviale cristallise. A quoi se rapporte cette frontière naturelle portée par le fleuve : une coupure -l'obstacle- ou une couture -ce cours d'eau libre de navigation?

\section{Le fleuve, une bonne frontière naturelle?}

Raisonner sur le fleuve comme "bonne » frontière " naturelle » au début du $\mathrm{XX}^{\mathrm{e}}$ siècle c'est emboiter deux débats sous une seule question bien laconique. Le premier renvoie à la distinction entre bonne et mauvaise frontière, qui prend la tournure d'un jugement de valeur. Le second débat distingue la frontière naturelle de la frontière artificielle et s'appuie sur les éléments de la géographie physique, que la Nature laisserait à la disposition de l'Homme. Ces deux débats sont placés sur le devant de la scène lors des remaniements frontaliers en Europe à la sortie de la Grande guerre. La rhétorique de la 
frontière naturelle appartient notamment à l'abondante littérature des revendications territoriales présentées par les délégations des pays. En outre, tous ces États en gestation aspirent à de bonnes frontières.

L'idée de frontière naturelle s'appuie sur un objet de nature qui relève de l'évidence physique, qui s'inscrit dans la longue durée, bref qui syncrétise le principe d'intangibilité dans le temps et dans l'espace: il est impossible de déplacer une montagne et la frontière sur sa ligne de crête ! Cette formulation qui relève du bon sens commun trouve vite ses limites quand il s'agit d'un fleuve. Le thalweg, repère frontalier communément admis, forme une ligne unissant les points les plus bas du cours du fleuve, mais il varie au gré de l'alimentation en eaux et de la rugosité des reliefs. Le cours d'eau se trouve modifié comme en témoigne la nouvelle de Yordan Raditchkov citée en exergue. S'appuyant sur un élément naturel fluctuant, la frontière est donc elle aussi soumise à modification.

Plus encore, comme le note Camille Vallaux, la recherche de la frontière naturelle relève d'un autre âge: "les frontières naturelles répondent à l'antique notion de la frontière comme espace vide: elles ne répondent pas aux définitions des lignes et des zones de démarcation nécessaires aux États ${ }^{8} »$. La divagation du lit fluvial ou la ligne de crête acérée forment des zones périphériques qui menacent par le vide démographique voire juridique le territoire de l'État moderne. La frontière naturelle correspond mal à la territorialité de l'État moderne et à sa maîtrise exhaustive d'un territoire borné. Dans le cas du fleuve, on est même placé en face d'une contradiction géographique: la frontière naturelle, espace du vide, s'accorde mal aux caractères des fleuves européens, qui attirent villes, industries et fortes densités humaines.

La frontière est ainsi conçue comme un équilibre momentané de forces, un «isobare politique ${ }^{9}$ " selon les termes de Jacques Ancel, autre géographe, acteur et témoin du conflit mondial. Elle ne s'impose donc pas pour des raisons intrinsèques de qualité, tels que les supports naturels : la querelle frontière naturelle versus frontière artificielle tombe. Le débat bonne et mauvaise frontière perd aussi de son sens dans le champ scientifique. Le type de support frontalier ne permet en aucune façon de mesurer la vertu politique de la frontière comme l'illustre l'ambiguïté du fleuve-frontière. D'ailleurs, le Danube comme support frontalier n'est pas placé au cœur de l'argumentation des traceurs de frontières en 1919-1920.

\section{Rhin et Danube : lectures du « fleuve mondial »}

A rebours même de l'idée de frontière naturelle séparatrice et porteuse de vide, les experts du Comité d'études s'appuient sur une vision du fleuve en tant qu'axe économique international. Emmanuel de Martonne emploie le terme de «fleuve mondial » à propos du Rhin pour caractériser son trafic connecté à la circulation mondiale: "le courant commercial qui circule sur le Rhin s'alimente à des sources lointaines : le minerai de fer vient de Suède et d'Espagne, le blé de Russie, de Roumanie des Etats-Unis ${ }^{10}$. » A l'appui de sa démonstration, le géographe fait référence à dix planches cartographiques ${ }^{11}$, malheureusement absentes du volume et de l'atlas, sauf une. Dans les titres de ces cartes, on note l'importance accordée aux faits de la circulation et des échanges sur et par le Rhin, véritable artère commerçante, industrielle et urbaine. Dans ce trafic international, le Rhin est mieux pourvu par sa situation que le Danube : 
«Notons d'abord le fait que le Rhin débouche sur une mer bordière de l'océan Atlantique, à l'origine des plus grands courants du commerce maritime mondial. Le Danube débouchant sur une mer intérieure, qui communique difficilement avec la Méditerranée elle-même a une situation singulièrement moins avantageuse ${ }^{12}$ "

L'argumentaire du géographe s'appuie ainsi sur des notions et des outils propres à son champ disciplinaire. L'analyse en terme de situation tout d'abord renvoie à la mise en perspective du fleuve dans ses relations avec d'autres lieux. Ce n'est donc pas le fleuve dans ses caractéristiques hydrologiques qui importe, - elles sont d'ailleurs brièvement présentées- mais bien les liens qu'il permet avec de plus vastes horizons que les simples régions qu'ils traversent. L'appareil cartographique, outil essentiel à la démonstration, permet de moduler les échelles d'analyse et d'insister sur les relations du fleuve avec les espaces environnants. Le Rhin est tour à tour envisagé dans ses effets spatiaux en Alsace-Lorraine, l'analyse des réseaux de canaux et leur trafic s'exprime à l'échelle européenne, enfin la navigation maritime rhénane est envisagée dans ses relations au monde. Le Rhin se fait axe d'échange plus que lieu de rupture. C'est la vision développée dans La France de l'Est par Paul Vidal de la Blache, vice-président du comité d'études jusqu'en 1918, année de sa mort.

La vision du Danube relève de la même conception du fleuve comme artère économique et commerciale essentielle. La commission européenne du Danube, créée en 1856, institue un régime de navigation internationale sur le cours inférieur du fleuve. Elle est présentée comme un modèle à reproduire pour le Rhin :

«ce qu'il faut c'est instituer une Commission internationale permanente ayant, comme la Commission danubienne, le caractère d'une puissance souveraine, avec son pavillon, son budget autonome, exerçant un contrôle absolu sur la navigation et les travaux qui l'intéressent jusqu'au lac de Constance ${ }^{13}$. ».

Mais ne nous trompons pas, ces expertises sont aussi empreintes d'une prise de position patriotique dissimulée sous le savoir-faire de l'universitaire. Développer la conception d'un fleuve international, c'est aussi contrecarrer pour les Français les ambitions des puissances centrales: d'un côté, les arguments nationalistes allemands du Rhin fleuve d'Allemagne et non frontière d'Allemagne ${ }^{14}$; de l'autre, les prétentions austro-hongroises d'accroître leur emprise sur la voie danubienne. Dans son plaidoyer pour une navigation rhénane internationale, Emmanuel de Martonne met habilement en valeur cet « intérêt vital » pour développer le port de Strasbourg, dans l'attente de son retour dans le giron français. Devant les autres délégations de l'Entente, l'argumentation en faveur de la liberté de navigation est assurée d'un certain succès. Elle traduit en effet une heureuse convergence des points de vue des puissances alliées puisque cette liberté peut s'entendre aussi bien dans son sens libéral, la liberté de commerce défendue par les Anglo-Saxons, que dans son sens révolutionnaire, la liberté des nations chère aux Français.

Quelle conception de la frontière fluviale révèle cet attachement à l'internationalisation de la navigation? Le terme de frontière est rarement prononcé dans ces études puisqu'il s'agit d'envisager les fleuves européens comme des systèmes spatiaux ouverts, porteurs d'échanges et non comme des limites étatiques. En creux, la question fluviale révèle un débat essentiel sur la définition juridique de la frontière. Deux conceptions s'opposent en ce début de $\mathrm{XX}^{\mathrm{e}}$ siècle et les experts du comité d'études en ont conscience. La "méthode diplomatique ${ }^{15}$ ", celle mise en pratique jusqu'alors, entérine des états de faits entre puissances et donc traduit des rapports de force : Le fleuve est partagé entre pays riverains selon une vision exclusive de l'appartenance 
territoriale. On retrouve ici l'argumentation de F. Ratzel. Quelques années plus tard, cette même vision alimentera la géopolitique de Karl Haushofer et sa conception de « l'État fluvial » selon laquelle le bassin du Rhin doit revenir en entier à l'Allemagne. Le fleuve est frontière séparatrice de territoires ou bien propriété exclusive d'un État. La "méthode juridique", nouvelle dans sa formulation, s'appuie sur la reconnaissance d'un droit international qui prend force de loi dans les traités ratifiés par les parties contractantes. La définition du fleuve international prend dans cette conception sa raison d'être; le fleuve-frontière peut exister sur la carte mais sa gestion en condominium, en tant que bien collectif des nations, s'apparente au principe d'extraterritorialité. La conception du fleuve développée par les experts français se rattache à cette seconde définition.

\section{Identifier les fronts et tracer les frontières autour du Danube en 1919-1920}

En ces temps de guerre, la question militaire devrait prendre une place importante. Dans les publications du comité d'études, la part accordée à la dimension stratégique du fleuve est bien plus faible que celle dévolue au statut international des grands fleuves européens. Et pourtant, le terme de limes vient à l'esprit dès que l'on raisonne sur le Rhin et sur le Danube. Il renvoie à cette zone-frontière entre l'Empire romain et les Barbares, un dispositif militaire qui court des bouches du Rhin à celles du Danube avec un segment fortifié non fluvial, depuis les environs de l'actuelle Coblence sur le Rhin à quelques kilomètres en amont de Ratisbonne sur le Danube ${ }^{16}$. Tactiques et armements ayant grandement changé en 1919, la question stratégique du fleuve prend une autre dimension :

«L'importance d'un fleuve comme frontière militaire résulte surtout de la direction de son cours, par rapport au front suivant lequel doit se faire le déploiement stratégique des armées d'attaque ${ }^{17}$. »

Ce qui apparait crucial, c'est le mouvement des armées : la frontière militaire marque un temps dans la progression des armées mais elle n'est pas une garantie absolue, elle reste cet "isobare " qui enregistre les fluctuations du front militaire. Le Général Bourgeois concède d'ailleurs que la progression de l'aviation dans la stratégie militaire remet en question la barrière naturelle que formerait le fleuve. Le fleuve ne forme donc pas forcément une frontière militaire recevable. Plus encore, la frontière stratégique apparaît comme non superposable à la frontière politique car elle obéit à des logiques différentes. La fixité et la linéarité de la frontière de l'État moderne s'accommodent mal de la marche militaire fluctuant pour contenir l'ennemi.

D'autres fronts viennent couturer l'espace balkanique. A l'image du limes entre Empire romain et Barbares, les confins entre Empire ottoman, Empire austro-hongrois et Empire russe sont marqués par des marches militaires. A cela s'ajoutent les aires d'extension et de contact entre christianisme occidental, christianisme oriental et islam. Les aires linguistiques différentes viennent se superposer de façon non concordante à ces dernières ${ }^{18}$. Tous ces termes, limes, zone, marche, aires renvoient à l'idée de limites introuvables et mettent en exergue une dimension irréductible de l'espace, celle de sa continuité. Or, la frontière politique que les experts étudient incite au contraire à découper l'espace, à le démarquer selon des lignes identifiables. La frontière dans sa définition juridique est un marqueur de discontinuité spatiale, celle du ressort de deux souverainetés étatiques distinctes. Sur le terrain, elle est 
matérialisée par des postes-frontières et des bornes frontalières. Face aux indurations dans le temps et dans l'espace de limites floues à la linéarité introuvable, les membres du comité d'études doivent tracer des frontières linéaires et disjonctives, des « dyades " selon l'expression de M. Foucher pour caractériser une frontière commune à deux États contigus. On prend conscience ici de toute la difficulté de la tâche dans ces Balkans.

La tension entre continuité et discontinuité spatiale se double d'une autre difficulté : celle de dénombrer, de localiser et de catégoriser. C'est aussi tout l'enjeu de l'expertise : trouver des seuils pertinents pour raisonner non plus seulement sur du continu/ discontinu mais aussi sur de l'homogène/hétérogène. L'identification des composantes pour distinguer ce qui est homogène de ce qui est hétérogène fait partie de la tâche dans sa dimension scientifique. Ce qu'il faut dénombrer relève de peuples (Slaves du Sud ou du Nord, Latins, Magyars et Germains pour ne citer que les principaux), de langues (slaves, latines...), de religions du Livre et de nationalités (qui combinent différemment dans leur définition un critère linguistique fondé sur la langue maternelle ou la langue d'usage). Mais comment penser le recouvrement dans l'espace de ces zones de confins et l'hybridation de différentes catégories de différenciation des groupes humains quand il faut tracer une frontière et donc par définition départager? Une part du défi balkanique est ici.

L'autre part du défi des traceurs de frontières dans les Balkans réside dans l'aptitude à combiner ces caractères balkaniques endogènes aux critères exogènes formulés par les autorités diplomatiques de la Conférence de la Paix. De la proposition de frontière formulée au comité d'études à sa ratification dans les traités de paix issus de la Conférence de Paris, il y a loin. Et l'on sait depuis de nouvelles recherches d'historiens ${ }^{19}$ que l'avis des géographes experts a rarement été pris en considération dans les discussions diplomatiques pour tracer la frontière. Les critères principaux retenus pour déterminer les frontières sont bien connus: droits historiques, principe des nationalités, construction d'États nationaux reconnus par le droit international dans l'objectif de garantir la paix. Toute la difficulté est justement de faire sortir de la gangue territoriale des empires aux contours flous des États nationaux aux territoires bornés. Or, dans cet espace balkanique plus riche de nations que d'États, postuler que ces derniers stimulent la cohésion nationale et territoriale, à l'instar du modèle français, c'est oublier qu'ici les identifications nationales précèdent des constructions territoriales étatiques jeunes ou en gestation. En fait, le travail des experts sur les Balkans soulève la contradiction de la frontière de l'État national : comment envisager des limites de territoires étatiques discontinus et homogènes, alors qu'une multitude d'identités en solution de continuité spatiale mais hétérogènes les peuplent? On voit bien que cette question excède largement la réflexion sur le Danube comme frontière ainsi que le raisonnement en terme de frontières naturelles ou artificielles, bonnes ou mauvaises.

\section{Trois enjeux frontaliers autour du Danube dans les Balkans}

Jusqu'à présent nous avons mené une réflexion sur le Danube comme support de frontière à l'échelle de son cours européen, la petite échelle du géographe. L'approche dans le détail de contentieux frontaliers autour du Danube nous permet d'aborder maintenant l'échelle plus fine des tracés sur le terrain. Et l'on sait combien les logiques 
géographiques peuvent être différentes selon l'échelle à laquelle on se place. Le contexte historique a évidemment sa part dans l'explication. Le Danube ne constitue pas une frontière inédite dans les Balkans : de Belgrade jusqu'à l'amont de Tutrakan, il forme déjà une frontière inter-étatique en 1913. Le fleuve est aussi support de limites administratives internes au sein du royaume de Hongrie. Cette frontière est remise sur le métier à la Conférence de la paix de Paris selon la logique de la "paix des vainqueurs »: pour une Roumanie et une Serbie dans le camp des vainqueurs, une Hongrie et une Bulgarie rangées dans celui des vaincus. Les experts comme les plénipotentiaires se montrent plus favorables aux prétentions territoriales des deux premières.

Le Danube n'est pas toujours l'enjeu principal des questions frontalières même s'il en constitue le repère géographique essentiel. Telle est notre hypothèse principale. Cette frontière naturelle si visible sur les cartes, si commode à identifier dans les cabinets diplomatiques est peu sollicitée dans l'argumentaire des experts géographes. En croisant les regards des scientifiques sollicités pour démêler les enjeux territoriaux, c'est la notion de frontière à l'épreuve du terrain que nous cherchons à explorer. La confrontation entre géographes et historiens, mais aussi géographes français et serbe permet d'aller plus avant dans les notions et les savoir-faire mobilisés. Pour ce faire, on s'appuie sur les textes mais aussi sur les cartes produites.

\section{Encadré 1 : notices biographiques des experts cités}

\footnotetext{
Jovan Cvijic (1865-1927), géographe serbe, était spécialiste de géomorphologie du karst. Fondateur de l'institut de géographie de l'Université de Belgrade et de la société serbe de géographie, ses réseaux scientifiques s'étendaient de Vienne à Paris, villes où il a effectué des séjours de recherche. Ses travaux se sont peu à peu orientés vers la géographie humaine avec une longue série d'études sur les populations balkaniques pour l'Académie des sciences dont il fut président en fin de carrière.

Émile Haumant(1859-1942), titulaire de la chaire de littérature russe à la faculté de Paris, était diplômé de l'École des Chartes et de l'institut des langues orientales. Agrégé d'histoire et docteur ès lettres, il était spécialiste de la Russie et des pays slaves.

Emmanuel de Martonne (1873-1956), géographe français, a contribué à consolider la géographie française dans le champ des disciplines universitaires, dans la lignée de son beau-père Paul Vidal de la Blache. Spécialiste de géographie physique, il fut titulaire de la chaire de géographie à la faculté des lettres de Paris. Homme de terrain, il a parcouru l'Europe centrale et particulièrement la Roumanie pour nourrir sa réflexion théorique sur la géographie physique et régionale.

Paul Vidal de la Blache (1845-1918), géographe français, il est considéré comme un des fondateurs de l'école française de géographie. Historien de formation, il se consacre à la construction de la géographie comme discipline scientifique à l'ENS puis à la Sorbonne. Cofondateur des Annales de Géographie, il est aussi l'auteur de grands classiques comme un atlas ou des cartes murales à l'usage des écoliers français ou bien encore du Tableau de la géographie de la France qui ouvre L'histoire de France dirigée par E. Lavisse.
} 


\section{Les méandres de la " Mésopotamie » pannonienne : la frontière entre Yougo-Slavie et Hongrie}

La question de la frontière septentrionale de la Yougo-Slavie donne lieu à une abondante littérature. La frontière héritée entre la Serbie et le Royaume de Hongrie suit la Save puis le Danube à partir de Belgrade, plaçant cette ville en position vulnérable à quelques encablures d'une frontière placée en plaine. La Serbie, placée dans le camp des vainqueurs, peut prétendre repousser ses frontières au-delà du fleuve, reste à en justifier les limites. Nous avons étudié deux expertises de cette frontière : celle du géographe serbe Jovan Cvijic, au titre d'expert mandaté par ce pays, et celle d'Émile Haumant, historien des langues, en tant que membre du comité d'études français ${ }^{20}$. Le dialogue entre les deux hommes n'est pas nouveau et poursuit une connaissance réciproque des productions scientifiques ${ }^{21}$. Sur le fond, les deux interventions sont en accord, on connaît d'ailleurs le patriotisme attesté chez le premier, au point de prendre le surnom de Dinaricus, et le sentiment pro-slave, ici en faveur des Serbes, établi chez le second ${ }^{22}$. Il ne s'agit donc pas ici d'étudier deux argumentations contradictoires mais bien de s'interroger sur la valeur de l'argument $\mathrm{du}$ fleuve-frontière parmi les nombreux critères pour tracer la frontière.

L'enjeu principal de cette frontière septentrionale du royaume en gestation des Serbes, des Croates et des Slovènes ne réside pourtant pas dans l'interrogation sur la frontière fluviale mais concerne justement cette identité nationale des Slaves du Sud. La réflexion sur la nation -qu'est-ce qu'être yougo-slave ?- précède bien celle sur l'État et ses limites. Ou plus exactement la première conditionne l'existence de la seconde. Jovan Cvijic consacre ainsi vingt pages de son opuscule à la "frontière ethnographique » avant d'aborder dans les deux parties suivantes les "frontières géographiques", découplées en deux pages pour l'étude des fleuves du bassin pannonien et deux pages pour le karst et la question des frontières avec l'Italie. Pour lui,

«si l'on a à choisir entre les faits ethnographiques et les raisons géographiques et stratégiques, c'est le plus souvent l'ethnographie qui doit l'emporter [...]. Deux nations voisines s'entendront mieux et noueront certainement plus facilement des relations de bon voisinage, même avec de mauvaises frontières stratégiques, si ces frontières ne blessent pas leurs sentiments nationaux et n'entravent pas leur développement économique $[. . .]^{23} \ggg$.

L'historien français propose un tracé concret de la frontière mais concède que

"Cette frontière sera difficile à tracer. Elle ne peut correspondre à des limites politiques, ni s'appuyer sur des lignes d'eau ou des sommets ; rarement elle pourra suivre une démarcation tranchée entre deux zones linguistiques; partout il faudra transiger entre les conditions communément exigées d'une bonne frontière $»^{24}$.

L'argument de la frontière naturelle et les critères de la bonne frontière sont ainsi vite congédiés par les deux experts. La limite des peuples - ethnographique selon Cvijic - ou des langues - linguistique selon Haumant - apparaît comme le critère principal, les considérations d'ordre géographique ou stratégique sont placées au second plan.

La définition de la nation yougo-slave est donc en jeu: comment extraire de ces grands empires multinationaux ces multiples nationalités et les amalgamer dans un État national en construction autour du noyau du royaume de Serbie? Fort de ses travaux antérieurs, Cvijic construit une nation yougo-slave où les différences de langues, de religions ou d'alphabets ont été dépassées grâce à la cohésion introduite par les 
mobilités des hommes. De son côté, Haumant s'attache davantage au principe des nationalités et aux limites linguistiques.

Les migrations incessantes et nombreuses dans cette partie de la péninsule balkanique ont fait l'objet d'une étude approfondie par le géographe serbe, épaulé par de nombreux collaborateurs ${ }^{25}$. Définis par le terme grec de "métanastasiques", littéralement changements d'habitat, ces mouvements sont répertoriés soigneusement à la manière d'un « lever " topographique et géologique, comme le revendique l'auteur. Il s'agit aussi d'une enquête anthropologique qui s'appuie sur l'origine des familles. Ces migrations continues au cours des derniers siècles, à plus ou moins long rayon d'action, aux effectifs très variables recouvrent un caractère serbe. En cela, elles se différencient des migrations médiévales et des invasions plus récentes qui ont eu -par l'installation de colons et de leurs îlots de peuplement- un double effet: la désagrégation du peuplement slave et les tentatives d'assimilation des populations locales aux envahisseurs. Cvijic distingue ainsi quatre courants de migrations -dinarique, kossovar, vardarien, et des courants franchissant la Save et le Danube. Ce faisant, le géographe fait des mobilités du peuple serbe le moteur d'une unification nationale.

« Il en résulta d'abord un enchevêtrement des groupes et des religions ainsi qu'un déplacement des dialectes des régions dinariques et du centre de la Péninsule vers le Nord. En même temps que leur dialectes, les immigrants balkaniques communiquèrent à l'ancienne population leurs mœurs et leur folklore; ils lui apprirent leurs chants nationaux d'origine serbe et lui apportèrent la conscience nationale très développée qui s'était élaborée au cours des siècles dans les pays balkaniques $»^{26}$.

Par la confrontation à l'autre, le Turc, le Hongrois ou l'Autrichien, l'unité entre les Yougo-slaves se fait aussi progressivement autour de la lutte commune contre l'envahisseur.

On en revient alors aux questions du dénombrement, pour cerner l'homogénéité des groupes humains, et de la localisation, pour mettre en évidence les discontinuités spatiales entre ces groupes. La construction de groupes homogènes appelle la détermination de critères et de seuils numériques. Les critères communément convoqués, la langue, la religion, l'appartenance ethnique, sont différemment ordonnés par les deux experts.

Fin connaisseur des langues slaves, Haumant reconnaît très tôt que l'homogénéité par la langue est une gageure :

«En fait était de ce peuple quiconque parlait slave -nachki- jusqu'au point où il cessait d'être compris », et il ajoute plus loin «seulement, comme tous les Slaves des Balkans se comprennent à peu près en raison inverse de l'éloignement de leur lieu d'origine, le problème linguistique, à procéder ainsi, se résoudrait en une question de kilomètres. ${ }^{27}$.

La continuité de l'aire linguistique, difficile à scinder en sous-groupes, et l'hétérogénéité des dialectes sont délicates à combiner avec les principes du traceur de frontière: distinguer des groupes homogènes et faire passer la limite sur les discontinuités entre ces groupes ${ }^{28}$. Quant à la "culture", sous laquelle Haumant regroupe religions et alphabets, elle créerait un excès de limites supplémentaires. Quel critère adopté alors ? La religion serait un bon principe :

«En fait, il n'y a qu'un moyen, dans ses régions, d'arriver à la frontière satisfaisante : c'est d'y appliquer le principe des traités de Westphalie, cujus regio ejus religio, et de faire émigrer les groupes épars du côté où il ne devrait pas être $»^{29}$. 
Mais là encore, les pressions des États pourraient se substituer aux émigrations volontaires. Le droit des nationalités et celui des peuples à disposer d'eux-mêmes prévaut sous la plume de l'historien français, il s'inscrit ainsi dans la lignée de la définition de la nation d'Ernest Renan : "dans cette incertitude force est d'arriver au vrai criterium des nationalités, l'assentiment des peuples $»^{30}$. Mais une fois de plus, avec l'exemple de la Macédoine, l'historien se trouve confronté aux différentes appellations, qu'elles résultent de dénominations locales ou étrangères. La frontière qu'il propose en 1918 résulte donc d'un compromis entre les revendications serbes dont il tient compte dans les grandes lignes et une nation serbo-croate qu'il appelle de ses vœux dans ses écrits, sans parvenir à en définir la cohérence interne.

Sous la plume de Cvijic, la défense des intérêts serbes s'appuie sur des travaux scientifiques abondants, non exempts toutefois de partis-pris. Du nord au sud du Bassin Pannonien, le géographe serbe distingue parmi le groupe des Slaves, les Slaves de la Grande Moravie (les Tchéco-Slovaques), les Slaves pannoniens et les Slaves balkaniques qui par un judicieux glissement de vocabulaire se transforment en Serbo-Croates balkaniques. La dénomination contribue à construire l'homogénéité du groupe des Slaves du Sud. La production de cette homogénéité ethnique passe aussi par la prédominance de l'ethnie, les Serbo-Croates balkaniques, sur la religion : en associant Serbes et Croates, la distinction entre orthodoxes et catholiques se trouve de la sorte minorée. La race commune, dite dinarique, renforce ce caractère commun. L'unité ethnique des Yougo-slaves est ainsi proclamée, la différence de dialectes, de genres de vie, est réduite à l'idée de types psychiques, déclinés en variétés ${ }^{31}$.

La construction de groupes homogènes trouve son achèvement dans la représentation cartographique. La carte permet la mise en espace de ces groupes et la mise en évidence des discontinuités spatiales. Outil par excellence du géographe, elle est censée posséder une objectivité scientifique indéniable. Mais elle constitue aussi un instrument d'aide à la décision pour les plénipotentiaires de la Conférence de la paix. L'impression visuelle de la carte est ainsi primordiale, comme le révèle le choix de Serbo-Croates au détriment de celui d'Illyriens dans les dénominations :

«Cette patrie, ils l'appelèrent l'Illyrie; plus tard, en étudiant leur histoire [...] ils vinrent au mot composé «serbo-croate», et c'est peut-être dommage: illyrien, comme helvète ou belge, effaçait les divisions du passé, l'autre mot n'est un progrès que par sa précision relative. On ne voyait pas les Illyriens sur la carte ; on y trouve les Serbes et les Croates et leurs frontières, ou à peu près $\|^{32}$.

Les deux experts se sont livrés à une critique méthodique des statistiques issues des recensements hongrois pour produire une carte.

Figure 1 - Extraits de la carte de Jovan Cvijic, «Frontière septentrionale des Yougoslaves » 

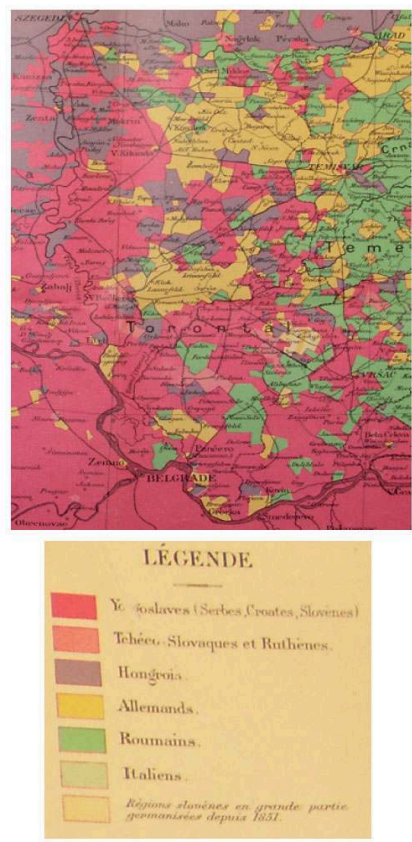

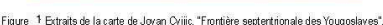

Figure 2 - Détails de la carte d'Emile Haumant, "Carte de la frontière septentrionale des pays yougoslaves ", dressée par le Service géographique des armées.
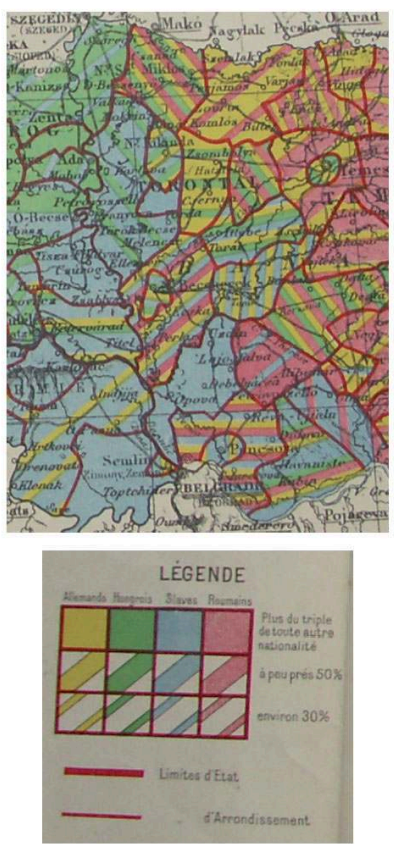

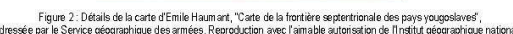

Encadré 2 : comparaison des cartes citées

\begin{tabular}{|l|l|l|l|}
\hline Figure & Figure 1 & Figure 2 & Figure 3 \\
\hline
\end{tabular}




\begin{tabular}{|c|c|c|c|}
\hline Auteur & Jovan Cvijic & Émile Haumant & $\begin{array}{l}\text { Emmanuel de } \\
\text { Martonne }\end{array}$ \\
\hline Source & $\begin{array}{l}\text { Frontière septentrionale } \\
\text { des Yougoslaves, Paris: } \\
\text { s.n. }\end{array}$ & \begin{tabular}{|lr} 
Travaux du & Comité \\
d'études, & Atlas \\
desQuestions & \\
européennes. & Paris: \\
imprimerie & \\
nationale. &
\end{tabular} & $\begin{array}{l}\text { Travaux du Comité } \\
\text { d'études, } \\
\text { desQuestions } \\
\text { européennes. Paris: } \\
\text { imprimerie nationale. }\end{array}$ \\
\hline $\begin{array}{l}\text { Titre de la } \\
\text { carte }\end{array}$ & $\begin{array}{l}\text { Carte ethnographique } \\
\text { des régions } \\
\text { septentrionales des } \\
\text { Yougoslaves }\end{array}$ & \begin{tabular}{|l} 
Carte de la frontière \\
septentrionale des \\
pays yougoslaves. \\
Répartition des \\
nationalités
\end{tabular} & $\begin{array}{l}\text { Répartition des } \\
\text { nationalités dans les } \\
\text { pays où dominent les } \\
\text { Roumains }\end{array}$ \\
\hline Date & 1919 & 1918 & 1919 \\
\hline Echelle & $1.000 .000^{\mathrm{e}}$ & $1.150 .000^{\mathrm{e}}$ & $1.000 .000^{\mathrm{e}}$ \\
\hline $\begin{array}{l}\text { Fonds de } \\
\text { carte }\end{array}$ & $\begin{array}{l}\text { Limites administratives } \\
\text { Cours d'eau principaux }\end{array}$ & $\begin{array}{l}\text { Carte d'état-major } \\
\text { avec relief et cours } \\
\text { d'eaux précis }\end{array}$ & $\begin{array}{l}\text { Limites } \\
\text { administratives } \\
\text { Cours d'eau principaux } \\
\text { Régions naturelles }\end{array}$ \\
\hline $\begin{array}{l}\text { Variable } \\
\text { qualitative }\end{array}$ & $\begin{array}{l}\text { Ethnies } \\
\text { (Yougoslaves, } \\
\text { Tchécoslovaques et } \\
\text { Ruthènes, Hongrois, } \\
\text { Allemands, Roumains, } \\
\text { Italiens) }\end{array}$ & $\begin{array}{l}\text { Nationalités. } \\
\text { (Slaves, Hongrois, } \\
\text { Allemands, } \\
\text { Roumains) }\end{array}$ & $\begin{array}{l}\text { Nationalités. } \\
\text { (Slaves, Bulgares, } \\
\text { Hongrois, Allemands, } \\
\text { Roumains, Turcs et } \\
\text { Tatares, Juifs) }\end{array}$ \\
\hline $\begin{array}{l}\text { Figuré de } \\
\text { couleurs }\end{array}$ & 6 & 4 & 7 \\
\hline $\begin{array}{l}\text { Variable } \\
\text { quantitative }\end{array}$ & Aucune & $\begin{array}{l}\text { Pourcentage } \\
\text { nationalités }\end{array}$ & densité \\
\hline $\begin{array}{l}\text { Nombre de } \\
\text { classes }\end{array}$ & Aucune & 3 & 3 \\
\hline
\end{tabular}

Chaque carte pose le problème du dénombrement des populations et de leur localisation dans l'espace. Sa lisibilité dépend en premier lieu du fonds cartographique adopté. Les éléments de la géographie physique sont limités aux cours d'eau sur la carte de Cvijic, alors que celle d'Haumant bénéficie du fonds physique détaillé fourni par le service géographique de l'armée, imprimeur du document. Cvijic privilégie les limites administratives : les comitat et leur subdivision en districts sont entièrement tracés sur sa carte alors que Haumant surimpose uniquement dans les zones contestées les limites des districts et comitat hongrois. Il en résulte une impression visuelle différente. Là où 
Haumant fait apparaître les effets de mixité entre les peuples dans le détail des circonscriptions administratives, la carte de Cvijic donne l'impression d'un patchwork : le géographe subdivise les territoires administratifs en aires de peuplement ethnique, sans fournir l'explication de cette subdivision. Ces aires peuvent recouvrir la dimension probable du finage des villages ou bien d'un groupement de communes. Sur aucune carte, la distinction du peuplement entre villes et campagnes n'est représentée.

En second lieu, le choix des variables, des figurés et des classes est déterminant dans l'impression visuelle donnée par la carte. L'historien français différencie dans la légende les populations selon quatre nationalités, -Allemands, Hongrois, Slaves et Roumains- et les discrétise en trois classes: les lieux où chaque nationalité est largement dominante, les lieux où elle est à égalité numérique avec une autre nationalité (par un figuré de larges hachures), les lieux où elle représente à peine un tiers de la population (figuré de fines hachures). C'est bien la représentation du seuil entre population minoritaire et majoritaire qui est recherchée ici. Chez Cvijic, la carte ethnographique rend compte de six groupes ethnographiques, les slaves étant subdivisés entre Yougoslaves (Serbes, Croates, Slovènes), d'une part, et TchécoSlovaques et Ruthènes, d'autre part, sans aucune information sur les effectifs ${ }^{33}$. Le but est de montrer dans quelle mesure les zones de colonies hongroises et allemandes viennent démembrer l'aire de peuplement des groupes slaves. De façon différente, les deux cartes montrent que les limites de peuplement ne sont pas tranchées, en revanche, le mode de représentation cartographique révèle un présupposé politique différent dans chaque cas. L'enchevêtrement des aires de peuplement doit conduire à l'autodétermination des peuples à disposer d'eux-mêmes chez Haumant, l'historien français évoque ainsi à plusieurs reprises la tenue de plébiscites ; aux yeux de Cvijic, il illustre l'usurpation territoriale au détriment des Serbo-Croates.

Quelle place accorder alors à l'argument du fleuve-frontière devant la primauté de l'argument ethnique? La frontière nord de la future Yougoslavie doit être déterminée dans la plaine pannonienne: ici pas de repère topographique marquant si ce n'est le Danube. Le fleuve n'est pas tant un enjeu frontalier qu'un point supplémentaire de légitimation en faveur d'une aire d'extension maximale du territoire yougoslave. Toute une série d'arguments plaide contre la fixation de la frontière sur le Danube aux yeux des deux experts.

Dans cette zone de flux et de reflux migratoires, l'argument historique de la préséance de tel ou tel peuplement est grandement malmené. Les deux experts notent que les envahisseurs ont fait peu de cas des grands cours d'eau car ceux-ci coulent dans le sens des vagues d'invasion : impossible alors de mobiliser le Danube et ses affluents comme un glacis protecteur. Cette zone de confins militaires entre Empire austro-hongrois et Empire ottoman, délaissée un temps par les politiques de colonisation, a connu un regain de magyarisation depuis la seconde moitié du XIX siècle, selon les dires du géographe serbe, contribuant à brouiller l'identification des populations locales.

En outre, les méandres divagants du fleuve forment une plaine fertile hydromorphe, comparée à juste titre à une "mésopotamie » par Haumant. Ces cours d'eau ont déjà porté des limites administratives au sein du royaume de Hongrie, en suivant la ligne du thalweg, une expérience peu concluante comme le note Cvijic.

«Le courant fluvial vient butter alternativement sur la rive gauche ou sur la rive droite. A l'endroit où se produit le choc, l'érosion est plus forte; elle fait reculer la rive et déplacer latéralement le thalweg. On voit, surtout dans les environs de Mohač et d'Apatin, de grandes boucles anciennes et d'autres plus récentes que le 
fleuve a recoupées. Le même phénomène s'observe sur la rive gauche de la Drave. Ainsi se sont formées de vastes plaines, parsemées de marais et de lacs riches en poissons, ainsi que de vastes îles entourées de bras morts, qui sont d'une grande fertilité.

Par suite du déplacement du Danube, la frontière administrative entre la Bačka et la Baranja n'est pas formée par le courant fluvial, mais par les méandres abandonnés. [...] $»^{34}$

Le tracé final est dans les mains des diplomates de la Conférence de Paris. La situation sur le front militaire dans les Balkans et la diligence avec laquelle les Slaves du Sud proclament leur union prennent de court les représentants des Alliés. La question yougoslave, abordée tardivement à Paris, connaît de multiples péripéties. Pour J. Bariéty, les frontières yougoslaves ne sont pas issues des expertises scientifiques, malgré la présence de Jovan Cvijic dans la délégation du royaume des Serbes, Croates et Slovènes ${ }^{35}$. Il affirme en revanche que les cartes ethnographiques ont été affichées dans un appartement du Quai d'Orsay mais il faut attendre plusieurs années pour que la frontière soit démarquée sur le terrain. L'argument ethnographique semble l'avoir emporté. L'argument économique en revanche est peu sollicité dans les propos des deux experts. Lucien Gallois le reproche vivement à Émile Haumant lors de la séance du comité d'études. Jovan Cvijic le mentionne succinctement à propos de Belgrade. On connaît pourtant la portée de cette question économique yougoslave : la baisse des flux économiques entre républiques fédérées est un signe précurseur du délitement du pays quelques dizaines de décennies plus tard...

\section{La question du Banat, entre Yougo-Slavie et Roumanie}

La détermination des frontières de la Roumanie, alliée des vainqueurs de la guerre et bénéficiaire d'un important "lobby roumain" en France, constitue une tâche importante de la Conférence de la Paix. Le comité d'études français, et plus particulièrement Emmanuel de Martonne, un de ses membres influents connu pour ses positions pro-roumaines, fournit de nombreuses expertises sur les limites à donner au futur territoire de la Roumanie. Le sort de plusieurs provinces, issues des grands empires et bordières du Danube et de ses affluents, est analysé par le géographe français : le Banat, entre Maros, Tisza et Danube, et la Dobroudja, sur les bouches du Danube, constituent d'épineuses questions frontalières. Entre les deux provinces, le long segment du Danube porte la frontière bulgaro-roumaine à la faveur d'une rive bulgare plus élevée que du côté roumain.

La question du Banat a fait déjà l'objet d'une étude approfondie dont nous nous contentons de reprendre ici les conclusions ${ }^{36}$. Devant la complexité ethnographique de la zone, le poids des villes et l'importance des problèmes de voies de communication, le Danube et son affluent, la Tisza, ne constituent pas les enjeux essentiels du tracé frontalier. Pour trouver un compromis entre les revendications serbes et roumaines, deux États alliés, le rôle d'E. de Martonne est primordial. Ses expertises sont érudites et très bien renseignées; son avis est sollicité par les commissions de diplomates à la Conférence de Paris. Le géographe présente une expertise favorable à la cause roumaine, dont il est un fervent défenseur. Son argumentation scientifique s'appuie sur son savoir-faire de géographe et sur deux atouts maîtres : la carte ethnographique et la notion de région.

La carte ethnographique révèle dans le Banat un véritable «manteau d'Arlequin » de nationalités, difficile alors d'y appliquer le principe des nationalités. La carte 
représente les nationalités présentes dans le Banat, selon trois classes de densité, selon leur proportion (système de hachures) et selon le lieu de peuplement, les villes sont l'objet d'un traitement spécial en diagramme circulaire. Elle a donné lieu à un travail minutieux de collecte et de recoupement de l'information statistique explicité dans un article des Annales de géographie ${ }^{37}$. Ce document a certes une valeur scientifique de représentation cartographique innovante mais il change aussi de statut: la carte n'est plus descriptive, elle devient un outil d'aide à la décision.

L'argument ethnographique révélant ici ses limites, le géographe convoque la notion de région pour montrer le tropisme du Banat vers les terres roumaines.

«L'opposition de la plaine et de la montagne reste aussi tranchée que possible dans le Banat, au point de vue physique, économique et ethnique. Mais il arrive souvent que deux régions très différentes sont, par là même solidaires; leurs ressources se complétant mutuellement $»^{38}$.

La région géographique est ainsi conçue comme un ensemble construit par les faits de complémentarité entre les différents types d'espaces et par les flux qui les animent. La région n'est donc pas seulement une unité physique homogène dans la définition de la région naturelle issue de la géographie physique, c'est aussi et surtout une construction fondée sur des solidarités humaines, économiques et politiques, constitutives d'un territoire. La cohésion interne du territoire régional l'emporte sur ses limites extérieures. On en comprend alors toute la portée pour cerner la notion de frontière en géographie. La frontière n'est pas envisagée comme une simple ligne politique, séparant des États, mais elle prend l'épaisseur d'une enveloppe qui distingue un territoire dont la cohésion et la cohérence internes sont plus fortes que les liens qu'il entretient avec d'autres ensembles territoriaux. Toute l'argumentation de de Martonne s'attache ainsi à construire un territoire roumain plus que son enveloppe externe. Il s'agit d'une territorialisation de la frontière qui s'appuie avant tout sur une territorialité d'ordre identitaire, celle qui créée des solidarités territoriales, et pas seulement sur une territorialité d'ordre politique, celle qui institue des limites juridiques d'État.

La force de l'argumentation du géographe lui confère une autorité scientifique mise à profit dans les délibérations de la Conférence de la Paix. De Martonne, carte à l'appui, propose un tracé de compromis entre les revendications serbes et roumaines d'une part, et les résolutions de la commission territoriale en charge de la question, d'autre part $^{39}$. Un accord est trouvé qui tempère les échanges de populations, garantit les voies de communication et donc les échanges; il est entériné par le traité de Trianon du 4 juin 1920 et ne sera pas remis en cause ultérieurement. Le Danube comme éventuel support de frontière a été exclu des discussions. 
Figure 3 - Détails de la carte d'Emmanuel de Martonne, "Répartition des nationalités dans les pays où dominent les Roumains dresssée par le Service géographique des armées »

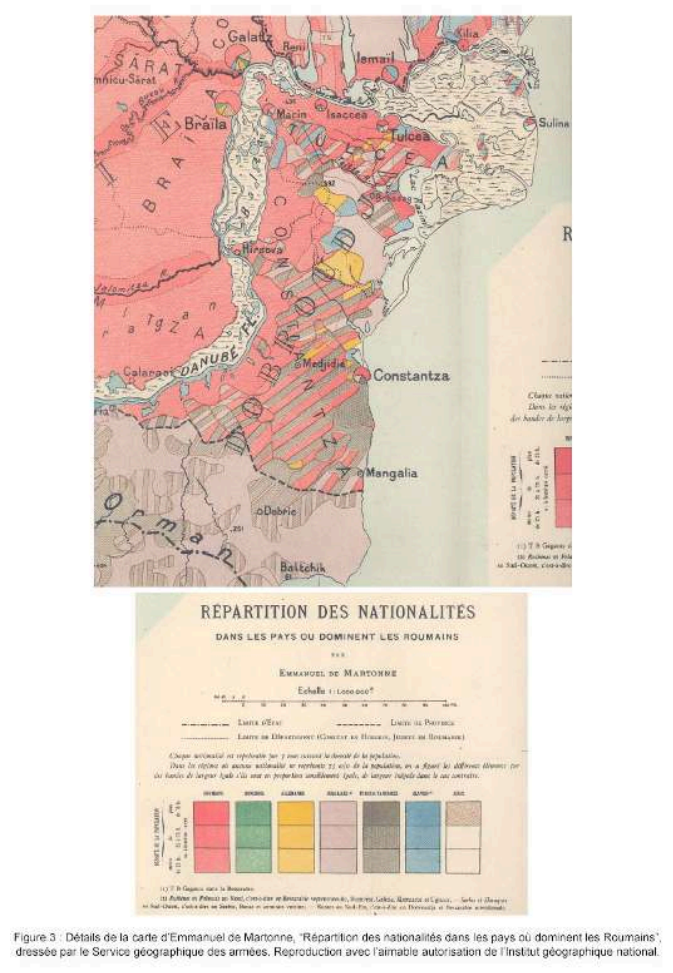

\section{La question de la Dobroudja et de la maîtrise des bouches du Danube}

La question de la Dobroudja est tout aussi complexe bien que fort différente. Comprise entre le coude que forme le Danube à partir de Silistra à l'ouest, les bouches du Danube au nord et la mer Noire à l'est, la Dobroudja n'a de limites incertaines qu'au sud, objet de conflits entre la Roumanie et la Bulgarie durant les Guerres Balkaniques puis la Première Guerre mondiale. Le « quadrilatère » de la Dobroudja, tel que le nomment les Roumains, ou Deli Orman (forêt folle) selon l'appellation turque qui est restée, constitue un bombement et une ligne de partage des eaux. Il correspond à la zone de fluctuation frontalière entre la frontière issue du Congrès de Berlin de 1878 et celle établie en 1913. Le devenir de cet espace est un enjeu majeur. Cependant, l'exposé d'Emmanuel de Martonne auprès du comité d'études excède cette simple question pour prévenir en fait toute revendication bulgare sur la Dobroudja en entier, jusqu'au delta du Danube. Il est vrai que le bruit des armes gronde encore dans la zone, rendant incertain l'issue du conflit. La question de la Dobroudja aura donc de toute évidence une issue militaire. Mais la roumanophilie de l'expert français est ici criante peut-être aussi parce que son argumentaire scientifique perd de sa force.

En effet, la Dobroudja, vaste plateau prolongé au nord par des collines, forme au sud une ancienne zone de forêts défrichée par les Turcs; elle devient un lieu de marécages à mesure que l'on se rapproche du Danube. Ce milieu plutôt hostile a connu des vicissitudes de peuplement : refuge de quelques minorités (tels les Lipovènes, Russes Vieux-croyants), lieu de passages des invasions qui y ont laissé des populations à la faveur d'une marche militaire, politiques de colonisations par la Roumanie notamment 
depuis la fin du XIX ${ }^{e}$ siècle. Le peuplement de la Dobroudja relève d'une histoire mouvementée, faite de flux et de reflux démographiques « où se mêlent confusément toutes les races connues sur les rivages de la mer Noire ». La notion de région si chère à la géographie française semble ici inopérante pour saisir ce mouvement chaotique de mobilités, à rebours des flux ordonnés en vue de créer une cohésion régionale. D'ailleurs de Martonne se garde bien de l'employer dans son texte au profit du terme plus neutre de " pays ».

Quels critères mobilisent donc le géographe pour accorder la Dobroudja et le «quadrilatère » à la Roumanie? Le paysage, tout d'abord, se rapproche de celui du Baragan roumain. On connaît l'aptitude de ce géographe a dessiné sous forme de croquis les paysages vus lors de ses nombreuses excursions de terrain ${ }^{40}$.

« Entre le Danube qui remontre vers le Nord et la mer Noire, les campagnes nues et sèches de la Dobroudja apparaissent comme la continuation des plateaux qui s'étalent au nord des Balkans, formées des mêmes couches crétacées non plissées, souvent recouvertes de tertiaire récent. Même absence d'eaux courantes sur de grandes étendues, mêmes villages très espacés. Pourtant l'aspect rappellerait plutôt les steppes russes que les plaines bulgares. [...] C'est au Baragan roumain, au sud de la Bessarabie que ressemble le plus la Dobroudja centrale et méridionale. ${ }^{41}$.

Le géographe procède ainsi par analogie avec une région roumaine et réfute de ce fait l'idée de région naturelle justifiée ici par la géologie. La région naturelle, fondée sur l'homogénéité paysagère, constitue pourtant une notion-clé de la géographie française de cette époque. Emportée par l'empathie pro-roumaine, l'argumentation scientifique fait ici entorse à la démarche géographique.

Si le terme de région n'apparaît pas clairement pour justifier la cohésion territoriale de la Dobroudja, le rôle de l'État roumain pour intégrer cet espace au territoire national est amplement souligné. C'est même ce qui justifie aux yeux de de Martonne la cession de la Dobroudja à la Roumanie. La politique de colonisation assure un développement agricole, une densification du peuplement et une modernisation des transports et des activités. Bref, par la colonisation, la Dobroudja est en passe de devenir une région organisée et intégrée à la Roumanie :« la Roumanie a le droit d'être fière des progrès réalisés en Dobroudja. D'un pays misérable, en grande partie turco-tatare, elle a fait un pays prospère, où la majorité roumaine est destinée à devenir de plus en plus grande $»^{42}$.

L'importance de cette majorité roumaine dans un espace pluri- ethnique ${ }^{43}$ pose cependant problème et il reste à démontrer son bien-fondé en s'appuyant sur l'argument reconnu du droit des nationalités. Le recoupement des différents recensements pratiqués dans la zone ne permet pas de faire ressortir une majorité roumaine écrasante, même si au fil des années (et surtout de la source quand elle roumaine!), l'élément roumain se renforce grâce à la colonisation. La "carte des nationalités dans les pays où dominent les Roumains" (figure 4) dressée par de Martonne montre un enchevêtrement de nationalités -Roumains, Bulgares, Turcs, Slaves, Allemands- avec notamment un noyau bulgare séparé de la mère-patrie. Le géographe convoque donc l'argument de la densité pour montrer que les Roumains constituent la population la plus peuplante dans ce front pionnier : « d'un pays désert, la Roumanie a fait une région où la densité de population atteint celle des États agricoles du centre américain». Le long du Danube notamment, la population roumaine est dense et largement dominante, ce qui conduit le géographe à conclure : "les régions ethnographiquement les plus mélangées ne sont pas les plus peuplées " ${ }^{44}$. 
Le Danube apparaît alors dans cette zone comme un axe de peuplement roumain solide sur ses deux rives.

L'intérêt géostratégique et économique du fleuve vient renforcer l'argument des nationalités en faveur d'une attribution de la zone à un pays allié :

« la cession de la Dobroudja à la Bulgarie signifierait, non seulement l'isolement de

la Roumanie et son asservissement, mais l'élimination de l'Entente de la

Commission danubienne, la mainmise des puissance centrales sur le Bas-Danube et

leur domination dans les Balkans $»^{45}$.

Pour protéger la nouvelle Roumanie, il faut en effet lui garantir un territoire facilement défendable. Or la Dobroudja est le lieu de passage de multiples invasions. Au sud, les hauteurs du Deli Orman permettent de fondre aisément sur la Roumanie : d'un point de vue stratégique, la Dobroudja doit donc être roumaine. Les raisons économiques de libre circulation sur le fleuve, défendue par les grandes puissances victorieuses, constituent enfin le dernier argument convoqué. Le géographe opère ici un changement d'échelle pour envisager les conséquences européennes néfastes d'une cession de la Dobroudja à la Bulgarie : la maîtrise des bouches du Danube permet celle des Balkans, les céder à la Bulgarie signifie les céder à l'ennemi. Le géographe se fait ici géopoliticien à la manière de son contemporain britannique, Halford J. Mackinder, dont la théorie du « heartland» fait de l'Europe centrale et balkanique le pivot géographique de l'Europe ${ }^{46}$.

Ce dernier argument est pris en considération par le Conseil supérieur des alliés à la Conférence de la Paix. Il décrète le statu quo ante arguant qu'une "modification de frontière [qui] entraînerait la cession à un État ennemi d'un territoire faisant en droit partie intégrante d'un État allié $\aleph^{47}$. La logique vainqueur/vaincu de la guerre l'emporte. Cela dit, cette frontière de diplomates sera de nouveau contestée après la Seconde Guerre mondiale. L'expertise d'Emmanuel de Martonne possède, dans le cas de la Dobroudja, une faible incidence. Elle présente cependant un intérêt pour l'histoire de la pensée géographique française car elle constitue un plaidoyer contre la frontière naturelle du Danube en Dobroudja: l'analyse du géographe, guidée aussi par son sentiment pro-roumain, conduit à céder le fleuve et son embouchure à la Roumanie.

\section{Conclusion}

Le Danube est-il un marqueur de frontière en 1919-1920 ? Il est repère spatial mais il n'est pas facteur prédominant du tracé. La carte continentale des frontières sur le Danube nous abuse ! A l'échelle locale, les multiples digressions de la frontière hors de la ligne fluviale marquent non pas une anomalie mais portent souvent la trace d'une mise en valeur séculaire qui a su s'affranchir de la barrière fluviale. La pertinence de la notion de frontière naturelle dépend donc étroitement de l'échelle d'observation. La frontière se trouve aussi souvent à l'articulation de systèmes territoriaux différents qui n'épousent pas forcément des éléments naturels. Elle peut être une cicatrice d'une cassure historique, les migrations spontanées ou forcées de populations après la Première Guerre mondiale nous le rappellent douloureusement pour les Balkans. Alors de quel sens sont porteuses ces frontières de 1919-1920 sur le Danube ? Leur remise en cause dans les conflits, leur volatilité marquée par le changement du tracé ne traduisent pas forcément de mauvaises frontières (même si elles se sont aux yeux de 
leurs détracteurs) mais elles expriment les dynamiques de systèmes territoriaux complexes.

En fait, les difficultés pour tracer la frontière dans les Balkans montrent le paradoxe de tout tracé frontalier d'un État-nation : s'agit-il de tracer des limites entre des États ou bien de déterminer des frontières entre les nations? Tant que le modèle de l'Étatnation dominera, la question sera en grande partie là. Et ce modèle que les géographes français ont en tête à la Conférence de Paris ne permet guère de raisonner en ces termes. Comme le résume $\mathrm{M}$. Foucher le modèle géopolitique français de cette époque se résume ainsi : "critique des fronts, éloge des frontières" car la frontière doit permettre de faire coïncider les bornes de l'État et l'enveloppe territoriale de la nation.

\section{NOTES}

1. Comme l'a montré notamment Monmonier (Mark), Comment faire mentir les cartes ou du mauvais usage de la géographie, Paris, Flammarion, 1993, $232 \mathrm{p}$.

2. Travaux du comité d'études, L'Alsace-Lorraine et la frontière du Nord-Est, Paris: Imprimerie nationale, 1918, 450 p. L'atlas correspondant ne contient en fait que des fac-similés de pétitions d'habitants des «pays rhénans " demandant à devenir citoyens français. Travaux du comité d'études, Questions européennes, Paris : Imprimerie nationale, 1919, 880 p. et Travaux du comité d'études, Atlas. Questions européennes, Paris : Service géographique de l'Armée, XXI planches.

3. "Conditions physiques et économiques de la navigation rhénane", in Travaux du comité d'études, L'Alsace-Lorraine et la frontière du Nord-Est, pp. 269-289.

4. "L'utilisation du Rhin comme force motrice », ibid., pp. 301-304.

5. « Neutralité et liberté de navigation du Rhin », ibid., pp. 245-263.

6. "Liberté et neutralité de la navigation du Danube", in Travaux du comité d'études, Questions européennes, pp. 665-682.

7. «Les variations de la frontière du Nord et du Nord-Est », in Travaux du comité d'études, L'Alsace-Lorraine et la frontière du Nord-Est, pp. 43-53.

8. Vallaux (Camille), Géographie sociale : le sol et l'État, Paris : O. Doin, 1911, p. 371.

9. Ancel (Jacques), Géographie des frontières, Paris : Gallimard, 1938, p. 194-195.

10. "Conditions physiques et économiques de la navigation rhénane », in Travaux du comité d'études, L'Alsace-Lorraine et la frontière du Nord-Est, p. 286.

11. La liste en est la suivante: planche $X$ : circulation commerciale en 1900 ; planche XI: circulation commerciale en 1912 ; planche XII : mouvement de la batellerie rhénane en 1912 ; planche XIII : batellerie rhénane, part des différents pavillons, 1912 ; planche XIV : circulation commerciale, courant du charbon; planche XV : circulation des minerais (fer essentiellement); planche XVI : circulation des fers ; planche XVII : circulation des céréales, planche XVIII : tonnage des principaux ports rhénans ; planche XIX : circulation sur les voies d'eau de l'Alsace-Lorraine et des pays voisins (carte en couleur en encart p. 272-273), «Conditions physiques et économiques de la navigation rhénane », in Travaux du comité d'études, L'Alsace-Lorraine et la frontière du Nord-Est, p. 269.

12. Ibid., p. 270.

13. Ibid., p. 286. 
14. Selon la formule d'Ernst Moritz Arndt en 1813 « Der Rhein- Deutschlands Strom, aber nichts Deutschlands Grenze ».

15. Selon la formule proposée par Charles Seignobos lors de la séance de clôture de la première série des travaux du Comité d'études, in Travaux du comité d'études, L'Alsace-Lorraine et la frontière du Nord-Est, p. 447 et suiv.

16. Beaupré (Nicolas), «Le Rhin : une géohistoire », La documentation photographique, 8044, 2005, 64 p. voir aussi Ayçoberry (Pierre) et Ferro (Marc) (dir.), Une histoire du Rhin, Paris : Ramsay, 1981, 459 p.

17. Général Émile Bourgeois, «Le Rhin, frontière militaire ", in Travaux du comité d'études, L'Alsace-Lorraine et la frontière du Nord-Est, p. 334.

18. L'ouvrage de Foucher (Michel), Fronts et frontières: un tout du monde géopolitique, Paris: Fayard, 1991 offre des cartes synthétiques de tous ces confins. Les travaux de Violette Rey actualisent le rôle de ces lignes de coupure dans l'Europe centrale et orientale après 1989, voir notamment Rey (Violette), «Feu l'Europe de l'Est?», Annales de Géographie, 1991, n555, pp. 564-578.

19. Nous renvoyons notamment à Bariéty (Jacques), «La Grande Guerre (1914-1918) et les géographes français », Relations internationales, 109, 2002, pp.7-24 ; Ter Minassian (Taline), «Les géographes français et la délimitation des frontières de la Bulgarie à la Conférence de la paix en 1919 », Balkanologie, VI (1-2), 2002, pp.199-212 et Ter Minassian (Taline), «Les géographes français et la délimitation des frontières balkaniques à la Conférence de la paix en 1919 ", Revue d'histoire moderne et contemporaine, 44 (2), 1997, pp.252-286. De notre côté, nous avons montré le rôle d'Emmanuel de Martonne dans le tracé de quelques segments de la frontière roumaine de l'expertise jusqu'au tracé final adopté in Boulineau (Emmanuelle), «Un géographe traceur de frontières : Emmanuel de Martonne et la Roumanie ", L'Espace géographique, 4, 2001, pp.368-369. en ligne à l'adresse suivante: http://www.cairn.info/article.php? ID_REVUE=EG\&ID_NUMPUBLIE=EG_304\&ID_ARTICLE=EG_304_0358

20. Cvijic (Jovan), Frontière septentrionale des Yougoslaves, Paris, s.n., 1919, 30 p. et 3 cartes h.t. et Haumant (Émile), «La frontière septentrionale de l'État yougo-slave », in Travaux du comité d'études, Questions européennes, Paris : Imprimerie nationale, 1919, pp. 533-543.

21. Le Français est un lecteur du géographe serbe dans le texte comme en témoigne sa note de lecture, Haumant (Émile), "Le pays dinarique et les types serbes d'après Mr Jovan Cvijic ", Annales de Géographie, 1914, 130, pp. 407-419 ainsi que «la nationalité serbo-croate ", Annales de Géographie, 1914, 127, pp. 45-59.

22. Cf. Bariéty (Jacques), «La France et la naissance du Royaume des Serbes, Croates et Slovènes, 1914-1919 », Relations internationales, 2000, 103, pp. 307-327.

23. Cvijic (Jovan), op.cit., p. 6.

24. Haumant (Emile), art.cit., p. 533.

25. L'édition complète des travaux de Jovan Cvijic a été publiée récemment par l'Académie des sciences serbe, pour une lecture en français voir Cvijic (Jovan), «Les mouvements métanastasiques dans la péninsule des Balkans ", Le monde slave, 1917, 1, $1^{\text {er }}$ juillet 1917, pp. 84-98 et Cvijic (Jovan), La péninsule balkanique, Paris : Colin, 1918, 529 p.

26. Cvijic (Jovan), Frontière septentrionale des Yougoslaves, Paris : s.n., 1919, p. 15.

27. Haumant (Emile), « La nationalité serbo-croate », Annales de Géographie, 1914, 127, pp. 50.

28. On s'appuie ici sur les travaux du linguiste Sériot (Patrick), « La linguistique spontanée des traceurs de frontière ", Cahiers de l'ILSL, nº, pp. 277-304.

29. Haumant (Émile), « La frontière septentrionale de l'État yougo-slave »..., p. 537.

30. Haumant (Emile), « La nationalité serbo-croate »...p. 52.

31. Et abondamment exposée dans Cvijic (Jovan), La péninsule balkanique, op. cit.

32. Haumant (Emile), « La nationalité serbo-croate »..., p. 47 
33. Les cartes de la Macédoine élaborées par J. Cvijic ont été remarquablement étudiées par Wilkinson (H.R.), Maps and Politics. A Review of the Ethnographic Cartography of Macedonia, Liverpool : University Press, 1951, 366 p.

34. Cvijic (Jovan), Frontière septentrionale des Yougoslaves ...p. 28.

35. Bariéty (Jacques), « La France et la naissance... », art.cit.

36. Voir Boulineau (Emmanuelle), « Un géographe traceur de frontière... », art.cit.

37. De Martonne (Emmanuel), «Essai de carte ethnographique des pays roumains ", Annales de Géographie, 1920, XXIX-158, pp. 81-98.

38. de Martonne (Emmanuel), »La question du Banat», in Travaux du comité d'études, Questions européennes, p. 563.

39. Archives du Ministère des affaires étrangères, Conférence de la Paix, Recueil des Actes, vol. 53, PV. 3, 6, 7 et 14 des mois de février et mars 1919.

40. On renvoie ici à Robic (Marie-Claire) et Joseph (Bernadette), "Autour des papiers de Martonne ", Acta geographica, 1987, 72, pp. 37-65, et à Robic (Marie-Claire), Baudelle (Guy) et Ozouf-Marignier (Marie-Vic), Géographes en pratique (1870-1945) : le terrain, le livre, la cité, Rennes : Presses universitaires de Rennes, 2001, 390 p.

41. de Martonne (Emmanuel), «La Dobroudja », in Travaux du comité d'études, Questions européennes, p.645.

42. ibidem, p. 654

43. Nous ne reprenons pas ici la présentation de l'histoire du peuplement de la Dobroudja détaillée par Ter Minassian (Taline), « les géographes français et la délimitation des frontières de la Bulgarie... », art.cit.

44. De Martonne (Emmanuel), « La Dobroudja »..., p. 652.

45. Ibidem, p. 657.

46. Mackinder (Halford J.), «The Geographical Pivot of History », Geographical Journal, 1904, 23, pp. 437-444.

47. Archives du Ministère des affaires étrangères, Conférence de la Paix, Recueil des Actes, vol. 53, annexe I.

\section{RÉSUMÉS}

La Conférence de la Paix de Paris en 1919, ouvre la question de remaniements ou de créations de frontières dans les Balkans. En s'appuyant sur les expertises des géographes mandatés par leurs gouvernements respectifs, on interrogera la place du Danube comme support de frontière au regard de la catégorie de "frontière naturelle » souvent débattue dans les travaux scientifiques et de la distinction entre «bonne » et " mauvaise » frontière. On montre que ces catégorisations tombent vite mais qu'apparaît un autre questionnement pour ces frontières balkaniques : il faut tracer une ligne continue qui départage des entités homogènes alors que les Balkans sont parcourus de fronts, ces zones discontinues sans homogénéité notable. On voit ainsi que la question du Danube comme porteur de frontière n'est pas l'enjeu essentiel et qu'il s'efface devant d'autres considérations. C'est du moins ce qui apparaît dans notre étude des cartes et des notions mobilisés par les géographes de l'époque. 
INDEX

Mots-clés : Carte, Front, Frontière, Région

Index géographique : Balkans, Bulgarie, Danube, Rhin, Roumanie, Yougoslavie

\section{AUTEUR}

\section{EMMANUELLE BOULINEAU}

Maître de conférences, ENS Lettres et Sciences humaines Lyon, Laboratoire Géophile- UMR 5600. emmanuelle.boulineau@ens-lsh.fr 